MATHEMATICS OF COMPUTATION

Volume 65, Number 216

October 1996, Pages 1431-1437

\title{
ULTRACONVERGENCE OF THE PATCH RECOVERY TECHNIQUE
}

\author{
ZHIMIN ZHANG
}

\begin{abstract}
The ultraconvergence property of a derivative recovery technique recently proposed by Zienkiewicz and Zhu is analyzed for two-point boundary value problems. Under certain regularity assumptions on the exact solution, it is shown that the convergence rate of the recovered derivative at an internal nodal point is two orders higher than the optimal global convergence rate when even-order finite element spaces and local uniform meshes are used.
\end{abstract}

\section{INTRODUCTION}

In the finite element history, there have been many investigations on derivative recovery techniques. For the literature, the reader is referred to [2] and references therein. Recently, Zienkiewicz and Zhu proposed a patch recovery procedure in which the recovered derivative is obtained by discrete least squares fitting on an element patch. What distinguishes this new recovery technique from the others is its practical effectiveness. The recovered derivative at an internal nodal point is simply the weighted average at some Gauss points in the adjacent elements. The computational cost for this postprocessing is almost free.

A surprising observation from numerical tests is that we can actually get an ultraconvergence (two orders higher) result based on the superconvergence (one order higher than usual) data. An $O\left(h^{4}\right)$ convergence rate has been reported for the recovered derivative at the internal nodal points when quadratic elements and uniform meshes are used. It has been conjectured that ultraconvergence will occur for any even-order finite element space (quadratic element has order 2). The current work is devoted to the theoretical justification of this astonishing phenomenon. We shall prove, for a certain class of two-point boundary value problems, that the recovery procedure will result in ultraconvergence internal nodal recovery when local uniform meshes and even-order elements are used.

\section{The PATCh ReCOVERY TEChNique}

Consider the following two-point boundary value problem:

$$
\begin{gathered}
-\left(a_{2}(x) u^{\prime}\right)^{\prime}-\left(a_{1}(x) u\right)^{\prime}+a_{0}(x) u=f \quad \text { in } I=(0,1), \\
u(0)=u(1)=0 .
\end{gathered}
$$

We assume that $a_{i}$ and $f$ are sufficiently smooth for our analysis. We also assume that $a_{2}(x) \geq \alpha>0$ for all $x \in \bar{I}$.

Received by the editor June 22, 1995 and, in revised form, November 2, 1995.

1991 Mathematics Subject Classification. Primary 65N30.

(C)1996 American Mathematical Society 
The weak formulation of (2.1), (2.2) is to find $u \in H_{0}^{1}(I)$ such that

$$
\left(a_{2} u^{\prime}, v^{\prime}\right)+\left(a_{1} u, v^{\prime}\right)+\left(a_{0} u, v\right)=(f, v), \quad \forall v \in H_{0}^{1}(I) .
$$

Let $\mathcal{T}_{h}, 0<h<1 / 2$, be a sequence of subdivisions of $\bar{I}$,

$$
\mathcal{T}_{h}=\left\{x_{i}\right\}_{i=0}^{N}, \quad 0=x_{0}<x_{1}<\cdots<x_{N}=1 ;
$$

denote $I_{i}=\left(x_{i-1}, x_{i}\right), h_{i}=x_{i}-x_{i-1}, h=\max _{i} h_{i}$, and set

$$
S_{h}=\left\{v \in H^{1}(I),\left.\quad v\right|_{I_{i}} \in P_{r}\left(I_{i}\right)\right\}, \quad S_{h}^{0}=\left\{v \in H_{0}^{1}(I),\left.\quad v\right|_{I_{i}} \in P_{r}\left(I_{i}\right)\right\} .
$$

We see that $S_{h}$ and $S_{h}^{0}$ are the spaces of continuous piecewise polynomials of degree not exceeding $r$ on $I$ under the subdivision $\mathcal{T}_{h}$.

The finite element solution of (2.3) is to find $u_{h} \in S_{h}^{0}$ such that

$$
\left(a_{2} u_{h}^{\prime}, v^{\prime}\right)+\left(a_{1} u_{h}, v^{\prime}\right)+\left(a_{0} u_{h}, v\right)=(f, v), \quad \forall v \in S_{h}^{0} .
$$

In order to define the recovered derivative, we introduce the Gauss points and the Lobatto points.

Let $L_{r}(x)$ be the Legendre polynomial of degree $r$ on $[-1,1]$. It is well known that $L_{r}(x)$ has $r$ zeros and $L_{r}^{\prime}(x)$ has $r-1$ zeros in $(-1,1)$. Denote by $g_{1}^{(r)}, \ldots, g_{r}^{(r)}$ the zeros of $L_{r}(x)$, and by $l_{1}^{(r)}, \ldots, l_{r-1}^{(r)}$ the zeros of $L_{r}^{\prime}(x)$ with $l_{0}^{(r)}=-1, l_{r}^{(r)}=1$; then $g_{j}^{(r)}, j=1, \ldots, r$, are called the Gauss points of order $r$, and $l_{j}^{(r)}, j=0,1, \ldots, r$, the Lobatto points of order $r$.

The Gauss and Lobatto points on $I_{i}$ are defined as the affine transformations of $g_{j}^{(r)}$ and $l_{j}^{(r)}$ to $I_{i}$, respectively:

$$
\begin{aligned}
& G_{i j}=\frac{1}{2}\left(x_{i-1}+x_{i}+h_{i} g_{j}^{(r)}\right), \quad j=1, \ldots, r, \\
& L_{i j}=\frac{1}{2}\left(x_{i-1}+x_{i}+h_{i} l_{j}^{(r)}\right), \quad j=0,1, \ldots, r .
\end{aligned}
$$

Here the index $r$ on $G_{i j}$ and $L_{i j}$ is dropped in order to simplify the notation.

In general, $u_{h}^{\prime}$ is a piecewise polynomial of degree $r-1$ and is discontinuous at the nodal points $x_{i}, 1 \leq i \leq N-1$. The recovered derivative by the patch recovery is a continuous piecewise polynomial of degree $r$ (as $u_{h}$ ), $R u_{h}^{\prime} \in S_{h}$, which is uniquely determined by its values at the Lobatto points. The values of the recovered derivative at the Lobatto points are obtained by the following least squares fitting procedure. On the element patch

$$
J_{i}=I_{i} \cup\left\{x_{i}\right\} \cup I_{i+1},
$$

consider a polynomial of degree $r$,

$$
p_{r}^{*}(x)=\left(1, x, \ldots, x^{r}\right) \boldsymbol{a} .
$$

The vector $\boldsymbol{a}=\left(a_{0}, a_{1}, \ldots, a_{r}\right)^{T}$ is computed by fitting, in the least squares sense, $u_{h}^{\prime}$ at $2 r$ Gauss points $\left\{G_{i j}, G_{i+1, j}\right\}_{j=1}^{r}$ in $J_{i}, i=1, \ldots, N-1$. Then the values of $R u_{h}^{\prime}$ at the Lobatto points are the values of $p_{r}^{*}$ at the same points, i.e.,

$$
\begin{gathered}
R u_{h}^{\prime}\left(L_{i j}\right)=p_{r}^{*}\left(L_{i j}\right), \quad j=1, \ldots, r \\
R u_{h}^{\prime}\left(L_{i+1, j}\right)=p_{r}^{*}\left(L_{i+1, j}\right), \quad j=0, \ldots, r-1 .
\end{gathered}
$$

Note that there is an overlapping of adjacent element patches, i.e., $J_{i-1} \cap J_{i}=I_{i}$, $1 \leq i \leq N-1$. If different patches result in different recoveries on $I_{i}$, an averaging 
is applied (see [3] for more details). But if the exact solution is a polynomial of degree $r+1$, we shall show that the two recoveries from adjacent patches are the same.

\section{UltraconVERGENCE ANALYSIS}

The first step of our analysis is to reduce (2.4) to a simpler problem. Subtracting (2.4) from (2.3) yields

$$
\left(a_{2}\left(u^{\prime}-u_{h}^{\prime}\right), v^{\prime}\right)+\left(a_{1}\left(u-u_{h}\right), v^{\prime}\right)+\left(a_{0}\left(u-u_{h}\right), v\right)=0, \quad \forall v \in S_{h}^{0} .
$$

Let $\tilde{u}_{h} \in S_{h}^{0}$ be given by

$$
\left(u^{\prime}-\tilde{u}_{h}^{\prime}, v^{\prime}\right)=0, \quad \forall v \in S_{h}^{0} .
$$

Then we have the following "superapproximation" and "ultra-approximation" results between $u_{h}$ and $\tilde{u}_{h}$ (see [2, Theorem 1.3.1 and Remark 1.3.1]):

Lemma 3.1. Let $u_{h}, \tilde{u}_{h}$ satisfy (3.1), (3.2), respectively. Then there exists a constant $C$, independent of $h$ and $u$, such that

$$
\left\|u_{h}^{\prime}-\tilde{u}_{h}^{\prime}\right\|_{L_{\infty}(I)} \leq C h^{r+1}\|u\|_{W_{\infty}^{r+1}(I)} .
$$

For the special case when $r \geq 2, a_{2}=1$, and $a_{1}=0$, we have

$$
\left\|u_{h}^{\prime}-\tilde{u}_{h}^{\prime}\right\|_{L_{\infty}(I)} \leq C h^{r+2}\|u\|_{W_{\infty}^{r+1}(I)} .
$$

By virtue of Lemma 3.1, we can reduce our discussion to a simple case:

$$
-u^{\prime \prime}=f \quad \text { in } I=(0,1), \quad u(0)=u(1)=0 ;
$$

or

$$
\left(u^{\prime}, v^{\prime}\right)=(f, v), \quad \forall v \in H_{0}^{1}(I),
$$

since the finite element solution of (3.5) satisfies (3.2).

In the following, we shall construct the finite element solution $u_{h} \in S_{h}^{0}$ for (3.5) and prove superconvergence and ultraconvergence properties of the recovered derivative.

We characterize $S_{h}^{0}$ by the following basis functions (cf. [1, p. 38]):

$$
S_{h}^{0}=\operatorname{Span}\left\{N_{i}(x), i=1,2, \ldots, N-1 ; \quad \phi_{j k}(x), j=1,2, \ldots, N, k=2,3, \ldots, r\right\} .
$$

Here,

$$
N_{i}(x)= \begin{cases}1+\left(x-x_{i}\right) / h_{i}, & x \in I_{i}, \\ 1+\left(x_{i}-x\right) / h_{i+1}, & x \in I_{i+1}, \\ 0, & \text { otherwise }\end{cases}
$$

is the usual finite element "tent" basis function, $\phi_{j k}$ is a "bubble" function with support on $I_{j}$ and its value on $I_{j}$ is defined as follows:

$$
\phi_{j k}(x)=\phi_{j k}\left(x_{j}-\frac{1-\xi}{2} h_{j}\right)=\phi_{k}(\xi), \quad \xi \in(-1,1),
$$

where

$$
\phi_{k}(\xi)=\sqrt{\frac{2 k-1}{2}} \int_{-1}^{\xi} L_{k-1}(t) d t
$$


and $L_{k-1}$ is the Legendre polynomial of degree $k-1$. Observe that

$$
\phi_{k}(-1)=\phi_{k}(1)=0, \quad \int_{-1}^{1} \phi_{k}^{\prime}(\xi) d \xi=0, \quad \int_{-1}^{1} \phi_{k}^{\prime}(\xi) \phi_{l}^{\prime}(\xi) d \xi=0, \quad k \neq l .
$$

We then have,

$$
\begin{gathered}
\phi_{j k}\left(x_{j-1}\right)=\phi_{j k}\left(x_{j}\right)=0, \quad \int_{0}^{1} N_{i}^{\prime}(x) \phi_{j k}^{\prime}(x) d x=0, \\
\int_{0}^{1} \phi_{j k}^{\prime}(x) \phi_{i l}^{\prime}(x) d x=0, \quad i \neq j \text { or } k \neq l .
\end{gathered}
$$

These orthogonality properties greatly simplify our analysis. We are able to express explicitly the finite element solution of (3.5) on $I_{i}$ as

$$
u_{h}(x)=u\left(x_{i-1}\right) N_{i-1}(x)+u\left(x_{i}\right) N_{i}(x)+\sum_{k=2}^{r} c_{i k} \phi_{i k}(x),
$$

where

$$
c_{i k}=\left(f, \phi_{i k}\right) /\left(\phi_{i k}^{\prime}, \phi_{i k}^{\prime}\right) .
$$

Theorem 3.1. Let $u$ be the solution of (3.5), and let $u_{h}$ be its finite element approximation on $S_{h}^{0}$. Assume that $u$ is a polynomial of degree not greater than $r+1$ on an element patch $J_{i}=\left(x_{i-1}, x_{i+1}\right)$. Then $R u_{h}^{\prime}=u^{\prime}$ on $J_{i}$.

Proof. From (3.6), we have, on $I_{i}$,

$$
u_{h}^{\prime}(x)=c_{i 1}+\sum_{k=2}^{r} c_{i k} \phi_{i k}^{\prime}(x)
$$

where

$$
c_{i 1}=\left(u^{\prime}, \chi_{I_{i}}\right) /\left(\chi_{I_{i}}, \chi_{I_{i}}\right)=\frac{u\left(x_{i}\right)-u\left(x_{i-1}\right)}{h_{i}},
$$

and $\chi_{I_{i}}$ is the characteristic function of $I_{i}$. By the definition of $\phi_{i k}$, we see that

$$
\operatorname{Span}\left\{\chi_{I_{i}}(x), \phi_{i k}^{\prime}(x), k=2, \ldots, r+1\right\}=P_{r}\left(I_{i}\right) .
$$

When $u \in P_{r+1}\left(J_{i}\right)$, we have $u^{\prime} \in P_{r}\left(I_{i}\right)$, and therefore

$$
\begin{aligned}
u^{\prime}(x) & =c_{i 1}+\sum_{k=2}^{r+1} \frac{\left(u^{\prime}, \phi_{i k}^{\prime}\right)}{\left(\phi_{i k}^{\prime}, \phi_{i k}^{\prime}\right)} \phi_{i k}^{\prime} \\
& =c_{i 1}+\sum_{k=2}^{r+1} \frac{\left(-u^{\prime \prime}, \phi_{i k}\right)}{\left(\phi_{i k}^{\prime}, \phi_{i k}^{\prime}\right)} \phi_{i k}^{\prime}=u_{h}^{\prime}(x)+c_{i, r+1} \phi_{i, r+1}^{\prime}(x) .
\end{aligned}
$$

Note that $\phi_{i, r+1}^{\prime}(x)$ is linearly dependent on the $r$ th-degree Legendre polynomial on $I_{i}$; therefore, it vanishes at the $r$ Gauss points $g_{i k}$ of $I_{i}$; i.e., $\phi_{i, r+1}^{\prime}\left(g_{i k}\right)=0$, $k=1, \ldots, r$. Hence,

$$
u_{h}^{\prime}\left(g_{i k}\right)=u^{\prime}\left(g_{i k}\right), \quad k=1, \ldots, r .
$$

Applying the same argument on $I_{i+1}$, we have

$$
u_{h}^{\prime}\left(g_{i+1, k}\right)=u^{\prime}\left(g_{i+1, k}\right), \quad k=1, \ldots, r .
$$


Recall that $R u_{h}^{\prime}$ is a polynomial of degree $r$ on $J_{i}$ and fits $u^{\prime}$, a polynomial of the same degree, in a least squares sense at the $2 r(r \geq 1)$ Gauss points on the element patch $J_{i}$ (since $u_{h}^{\prime}$ equals $u^{\prime}$ at these points). Therefore, $R u_{h}^{\prime}=u^{\prime}$ on $J_{i}$.

A direct consequence of Theorem 3.1 is the following superconvergence property.

Theorem 3.2. Let $u$ be the solution of (2.3), and let $u_{h}$ be its finite element approximation on $S_{h}^{0}$. Then there exists a constant $C$, independent of $h$ and $u$, such that

$$
\left|u^{\prime}\left(x_{i}\right)-R u_{h}^{\prime}\left(x_{i}\right)\right| \leq C h^{r+1}\left(|u|_{W_{\infty}^{r+2}\left(J_{i}\right)}+\|u\|_{W_{\infty}^{r+1}(I)}\right) .
$$

For the special case $a_{2}=1$ and $a_{1}=a_{0}=0$, we have

$$
\left|u^{\prime}\left(x_{i}\right)-R u_{h}^{\prime}\left(x_{i}\right)\right| \leq C h^{r+1}|u|_{W_{\infty}^{r+2}\left(J_{i}\right)} .
$$

Proof. The proof of (3.12) follows from Theorem 3.1 and the standard argument by applying the Bramble-Hilbert Lemma. The proof of (3.11) follows from Lemma 3.1 and (3.12) for the special case.

Based on Theorem 3.1, we can further prove the ultraconvergence result.

Theorem 3.3. Let $u$ be the solution of (2.3) when $a_{2}=1$ and $a_{1}=0$, and let $u_{h}$ be its finite element approximation on $S_{h}^{0}$ with $r(\geq 2)$ an even number. If the two elements on the element patch $J_{i}$ have the same length, i.e., $h_{i}=h_{i+1}$, then there exists a constant $C$, independent of $h$ and $u$, such that

$$
\left|u^{\prime}\left(x_{i}\right)-R u_{h}^{\prime}\left(x_{i}\right)\right| \leq C h^{r+2}\left(|u|_{W_{\infty}^{r+3}\left(J_{i}\right)}+\|u\|_{W_{\infty}^{r+1}(I)}\right) .
$$

Assuming further that $a_{0}=0$, we have

$$
\left|u^{\prime}\left(x_{i}\right)-R u_{h}^{\prime}\left(x_{i}\right)\right| \leq C h^{r+2}|u|_{W_{\infty}^{r+3}\left(J_{i}\right)} .
$$

Proof. We first prove (3.14). Associated with any interior node $x_{i}, i=1, \ldots, N-1$, there is an element patch $J_{i}=\left(x_{i}-h_{i}, x_{i}+h_{i}\right)$ (recall that $\left.h_{i}=h_{i+1}\right)$, and a linear mapping $F_{i}$ from $\hat{I}=(-1,1)$ onto $J_{i}$ defined by $x=x_{i}+h_{i} \xi$. Given any function $v$ on $J_{i}$, we define

$$
\hat{v}=v \circ F_{i}, \quad \text { or } \hat{v}(\xi)=v\left(F_{i}(\xi)\right)=v\left(x_{i}+h_{i} \xi\right) .
$$

Now, consider

$$
u^{\prime}\left(x_{i}\right)-R u_{h}^{\prime}\left(x_{i}\right)=\left\langle u^{\prime}-R u_{h}^{\prime}, \delta_{i}\right\rangle=h_{i}\left\langle\widehat{u^{\prime}-R} u_{h}^{\prime}, \hat{\delta}_{i}\right\rangle=h_{i} E\left(\hat{u^{\prime}}\right) .
$$

Here, $\hat{\delta}_{i}=\delta \circ F_{i}$ and $\delta$ is the discrete delta function. Obviously, $E\left(\hat{u}^{\prime}\right)$ is a linear functional which is bounded in $W_{\infty}^{r+2}(\hat{I})$. We shall show that $E\left(\hat{u}^{\prime}\right)$ vanishes when $\hat{u}^{\prime}$ is a polynomial of degree not greater than $r+1$.

Let $r=2 s$; we examine the case when

$$
u(x)=a\left(\frac{x-x_{i-1}}{h_{i}}\right)^{s+1}\left(\frac{x_{i+1}-x}{h_{i}}\right)^{s+1}, \quad a \neq 0,
$$

on $J_{i}$. Note that $u^{\prime}\left(x_{i}\right)=0$, and $u(x)$ is symmetric with respect to $x_{i}$ on $J_{i}$ (so is $u^{\prime \prime}(x)$ ). By definition, $\phi_{i k}$ and $\phi_{i+1, k}$ are symmetric (antisymmetric) with respect to $x_{i}$ when $k$ is even (odd). Therefore,

$$
\begin{gathered}
c_{i k}=\left(-u^{\prime \prime}, \phi_{i k}\right) /\left(\phi_{i k}^{\prime}, \phi_{i k}^{\prime}\right)=\left(-u^{\prime \prime}, \phi_{i+1, k}\right) /\left(\phi_{i+1, k}^{\prime}, \phi_{i+1, k}^{\prime}\right)=c_{i+1, k}, \quad k=2 l ; \\
c_{i k}=-c_{i+1, k}, \quad k=2 l-1 .
\end{gathered}
$$


Recalling (3.6), we have, on $J_{i}$,

$$
\begin{aligned}
& u_{h}(x)=a N_{i}(x)+ \begin{cases}\sum_{l=1}^{s} c_{i, 2 l} \phi_{i, 2 l}(x)+\sum_{l=1}^{s} c_{i, 2 l-1} \phi_{i, 2 l-1}(x), & x \in I_{i} \\
\sum_{l=1}^{s} c_{i, 2 l} \phi_{i+1,2 l}(x)-\sum_{l=1}^{s} c_{i, 2 l-1} \phi_{i+1,2 l-1}(x), & x \in I_{i+1},\end{cases} \\
& u_{h}^{\prime}(x)= \begin{cases}a / h_{i}+\sum_{l=1}^{s} c_{i, 2 l} \phi_{i, 2 l}^{\prime}(x)+\sum_{l=1}^{s} c_{i, 2 l-1} \phi_{i, 2 l-1}^{\prime}(x), & x \in I_{i} \\
-a / h_{i}+\sum_{l=1}^{s} c_{i, 2 l} \phi_{i+1,2 l}^{\prime}(x)-\sum_{l=1}^{s} c_{i, 2 l-1} \phi_{i+1,2 l-1}^{\prime}(x), & x \in I_{i+1} .\end{cases}
\end{aligned}
$$

Observe that

$\phi_{i, 2 l}^{\prime}\left(x_{i}-\tau\right)=-\phi_{i+1,2 l}^{\prime}\left(x_{i}+\tau\right), \quad \phi_{i, 2 l-1}^{\prime}\left(x_{i}-\tau\right)=\phi_{i+1,2 l-1}^{\prime}\left(x_{i}+\tau\right), \quad 0 \leq \tau \leq h_{i}$,

so that

$$
u_{h}^{\prime}\left(x_{i}-\tau\right)=-u_{h}^{\prime}\left(x_{i}+\tau\right), \quad 0 \leq \tau \leq h_{i} .
$$

By the patch recovery procedure,

$$
R u_{h}^{\prime}\left(x_{i}\right)=\sum_{j=1}^{r} \alpha_{j}\left[u_{h}^{\prime}\left(G_{i j}\right)+u_{h}^{\prime}\left(G_{i+1, r-j+1}\right)\right],
$$

where the $\alpha_{j}$ 's are weights of the least squares fitting. Note that when $h_{i}=h_{i+1}$, the Gauss points and weights are distributed symmetrically on $J_{i}$ with respect to $x_{i}$. By symmetry, we see that $x_{i}-G_{i j}=G_{i+1, r-j+1}-x_{i}$, and we set this value as $\tau$ in (3.17) to obtain

$$
u_{h}^{\prime}\left(G_{i j}\right)=-u_{h}^{\prime}\left(G_{i+1, r-j+1}\right) .
$$

We then have from $(3.18)$

$$
R u_{h}^{\prime}\left(x_{i}\right)=0=u^{\prime}\left(x_{i}\right)
$$

when $u$ is given by (3.16).

Since any $u \in P_{r+2}\left(J_{i}\right)(r=2 s)$ can be decomposed into

$$
u(x)=a\left(\frac{x-x_{i-1}}{h}\right)^{s+1}\left(\frac{x_{i+1}-x}{h}\right)^{s+1}+w(x)
$$

for some $a \in R^{1}$ and $w \in P_{r+1}\left(J_{i}\right)$, from Theorem 3.1 and (3.19) we see that

$$
R u_{h}^{\prime}\left(x_{i}\right)=u^{\prime}\left(x_{i}\right) \quad \forall u \in P_{r+2}\left(J_{i}\right),
$$

i.e., the linear functional $E\left(\hat{u^{\prime}}\right)$ vanishes for all $\hat{u}^{\prime} \in P_{r+1}(\hat{I})$. Therefore, by the Bramble-Hilbert Lemma, we have

$$
\begin{aligned}
\left|E\left(\hat{u^{\prime}}\right)\right| & \leq C\left\|\hat{\delta}_{i}\right\|_{W_{1}^{0}(\hat{I})}\left|\hat{u}^{\prime}\right|_{W_{\infty}^{r+2}(\hat{I})} \\
& \leq C h^{-1}\left\|\delta_{i}\right\|_{W_{1}^{0}\left(J_{i}\right)} h^{r+2}\left|u^{\prime}\right|_{W_{\infty}^{r+2}\left(J_{i}\right)}=C h^{r+1}|u|_{W_{\infty}^{r+3}\left(J_{i}\right)} .
\end{aligned}
$$

Note that $\left\|\delta_{i}\right\|_{W_{1}^{0}\left(J_{i}\right)} \leq C(r)$, a constant depending on $r$ only. Combining (3.15) and (3.21), we obtain (3.14). Finally, (3.13) follows from (3.14) and Lemma 3.1.

Remark 3.1. The ultraconvergence recovery result is local with regard to the mesh. If we want the ultraconvergence recovery at the node $x_{i}$, we only need to use uniform meshes adjacent to $x_{i}$. 
Remark 3.2. The ultraconvergence recovery for the general case where $a_{1} \neq 0$ is not known since we have only the superapproximation result (3.3) instead of the ultra-approximation result (3.4) in general.

Remark 3.3. The generalization of the result to the higher-dimensional tensor product case is not straightforward.

\section{REFERENCES}

1. B. Szabó and I. Babuška, Finite Element Analysis, John Wiley \& Sons, New York, 1991. MR 93f: 73001

2. L.B. Wahlbin, Superconvergence in Galerkin Finite Element Methods, Lecture Notes in Mathematics, Vol. 1605, Springer, Berlin, 1995

3. O.C. Zienkiewicz and J.Z. Zhu, The superconvergence patch recovery and a posteriori error estimates. Part 1: The recovery technique, Internat. J. Numer. Meth. Eng. 33 (1992), 13311364. MR 93c:73098

Department of Mathematics, Texas Tech University, Lubbock, Texas 79409

E-mail address: zhang@ttmath.ttu.edu 